\title{
Different stoichiometric Ratios Realized in Energetic-Energetic Cocrystals Based on CL-20 and 4,5-MDNI: A Smart Strategy to Tune Performance
}

Yanwei Tan, ${ }^{\text {a }}$ Yucun Liu, ${ }^{\mathrm{b}}$ Haojing Wang, ${ }^{\mathrm{b}}$ Hongzhen Li, ${ }^{\mathrm{a}}$ Fude Nie, ${ }^{\mathrm{a}}$ Zongwei Yang, ${ }^{* a}$

a Institute of Chemical Materials, China Academy of Engineering Physics (CAEP), Mianyang 621900, China.

${ }^{b}$ School of Environment and Safety Engineering, North University of China, Taiyuan 030051, China.

\section{Supporting Information (SI)}

\section{Table of Contents}

SI 1. Single Crystal X-ray Diffraction (SXRD) of the Cocrystal 1

SI 2. Single Crystal X-ray Diffraction (SXRD) of the Cocrystal 2

SI 3. Molecular Configurations of CL-20 in the Cocrystal 1 and Cocrystal 2

SI 4. Detonation Property Calculations

SI 5. References 


\section{SI 1. Single Crystal X-ray Diffraction (SXRD) of cocrystal 1}

The single crystal X-ray diffraction data for cocrystal 1 were collected using a Bruker APEX-II-CCD diffractometer with graphite-monochromated $\mathrm{Mo}-\mathrm{K} \alpha$ radiation $(\lambda=0.71073 \AA)$ at $130 \mathrm{~K}$. The minimum asymmetric unit and hydrogen bonding interactions are given in Figure $\mathrm{S} 1$ and Table S1.

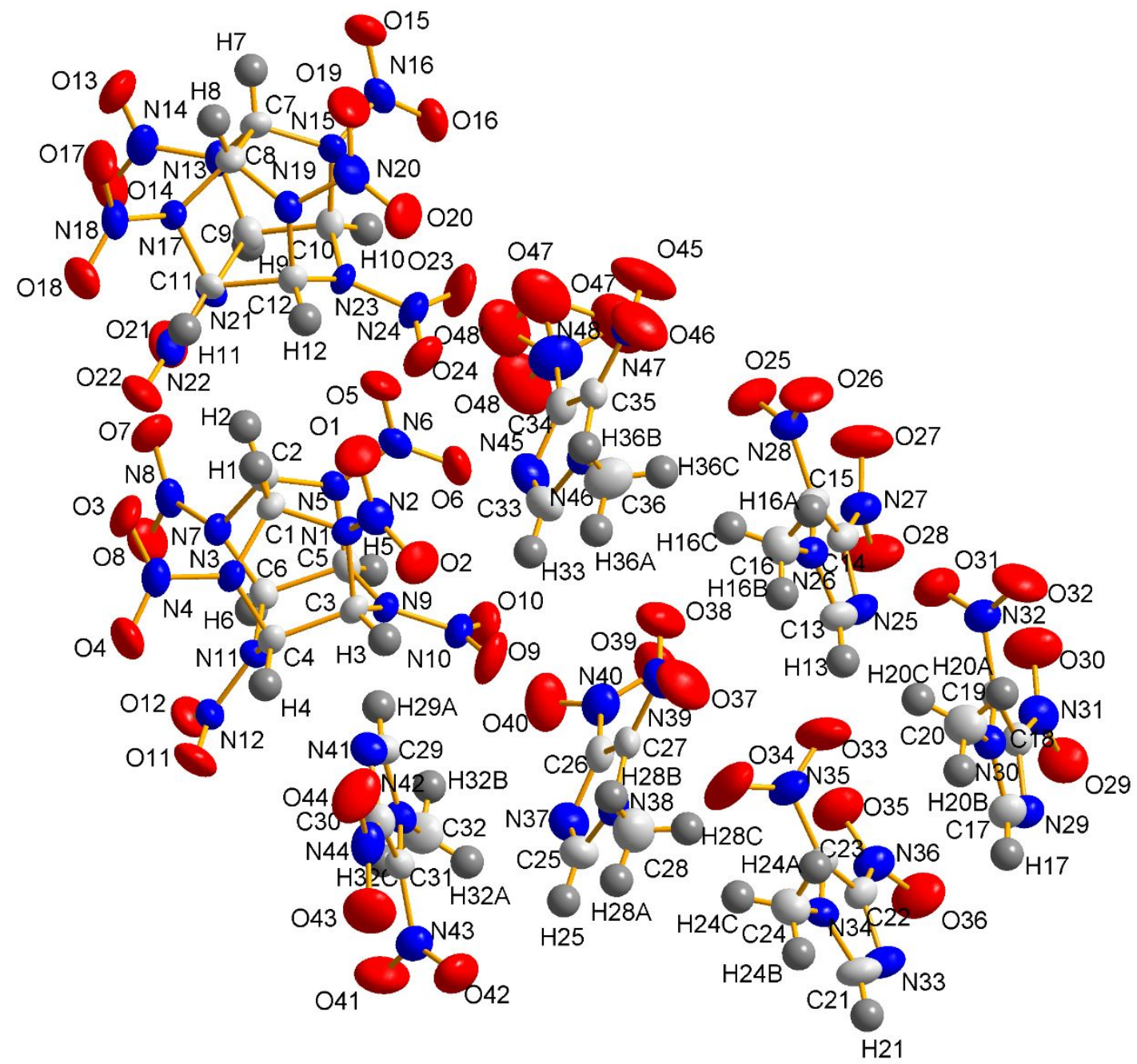

Figure S1. OPTEP diagram for cocrystal 1 with $50 \%$ probability ellipsoids 
Table S1. Bond lengths $(\AA)$ and angles $\left({ }^{\circ}\right)$ of hydrogen bonding interactions were found in cocrystal 1

\begin{tabular}{|c|c|c|}
\hline Interaction & Bong lengths $\AA$ & Bong angles $^{\circ}$ \\
\hline C34-H34...O28 & 2.4667 & 154.954 \\
\hline $\mathrm{C} 1-\mathrm{H} 1 \ldots \mathrm{O} 48$ & 2.3181 & 163.295 \\
\hline C12-H12...O48 & 2.7122 & 112.398 \\
\hline C36-H36A...O1 & 2.5482 & 142.543 \\
\hline C36-H36А...O21 & 2.6875 & 134.637 \\
\hline С32-H32B...O11 & 2.7746 & 141.885 \\
\hline C32-H32A...O1 & 2.7083 & 135.275 \\
\hline С6-H6...O40 & 2.3734 & 136.125 \\
\hline $\mathrm{C} 2-\mathrm{H} 2 \ldots \mathrm{O} 40$ & 2.71 & 122.468 \\
\hline C11-H11...O32 & 2.7817 & 113.338 \\
\hline C34-H34...O23 & 2.6874 & 117.663 \\
\hline С16-H16C...O19 & 2.6384 & 132.94 \\
\hline C10-H10...O27 & 2.3588 & 159.287 \\
\hline C20-H20...O8 & 2.3701 & 165.544 \\
\hline C7-H7...O32 & 2.3787 & 126.022 \\
\hline C3-H3...O31 & 2.789 & 101.141 \\
\hline C28-H28A...O18 & 2.6668 & 168.41 \\
\hline C9-H9...N27 & 2.201 & 166.516 \\
\hline C4-H4 ...N47 & 2.2366 & 152.72 \\
\hline
\end{tabular}




\section{SI 2. Single Crystal X-ray Diffraction (SXRD) of cocrystal 2}

The single crystal structure of cocrystal $2^{1}$ obtained from the CCDC, which was reported by Yang. The minimum asymmetric unit and crystal parameters including bond lengths and angles were listed in Figure S2 and Table S2. The main intermolecular interactions were exhibited in Figure S3.

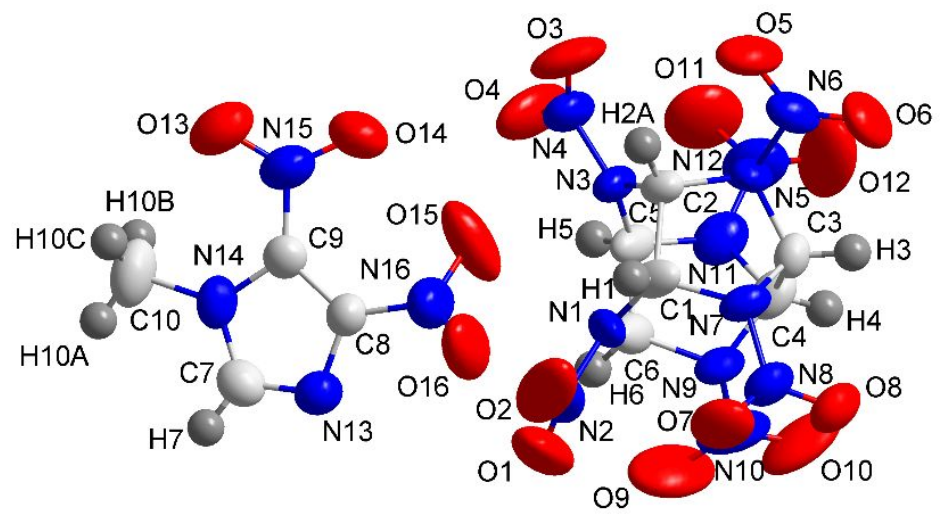

Figure S2. OPTEP diagram for cocrystal 2 with 50\% probability ellipsoids

Table S2. Bond lengths $(\AA)$ and angles $\left({ }^{\circ}\right)$ of hydrogen bonding interactions were found in cocrystal 2

\begin{tabular}{|c|c|c|}
\hline Interaction & Bong lengths $\AA$ & Bong angles $^{\circ}$ \\
\hline C6-H6...O16 & 2.5407 & 133.792 \\
\hline C9-H9...O4 & 2.5749 & 117.479 \\
\hline C3-H3...O15 & 2.57 & 128.247 \\
\hline C4-H4 ...O16 & 2.6445 & 140.512 \\
\hline C10-H10A...O1 & 2.5942 & 133.789 \\
\hline C10-H10C...O3 & 2.775 & 122.628 \\
\hline
\end{tabular}


(a)

(b)
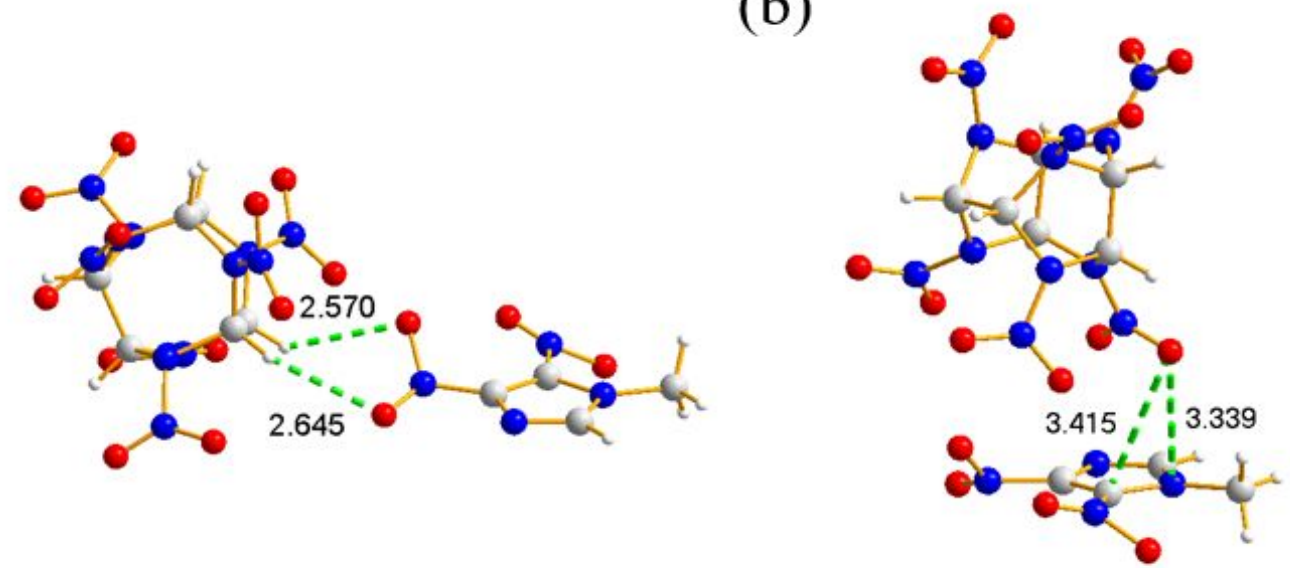

Figure S3. The main intermolecular interactions of the cocrystal 2 
SI 3. Molecular Configurations of CL-20 in the Cocrystal 1 and Cocrystal 2

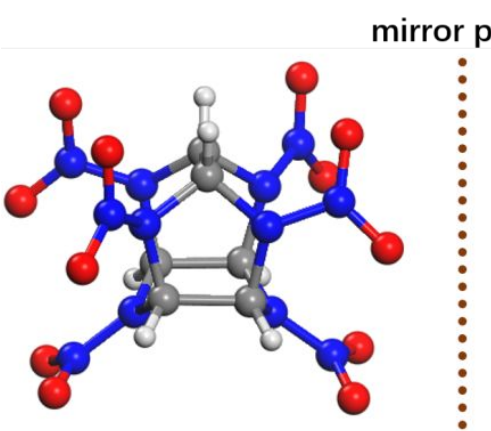

CL-20-a

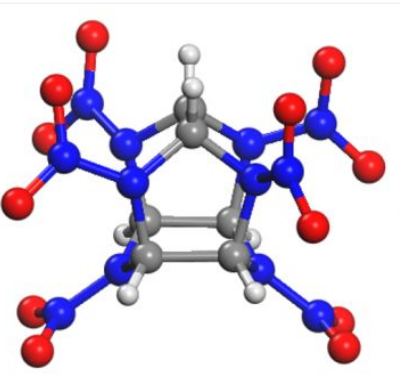

CL-20-b

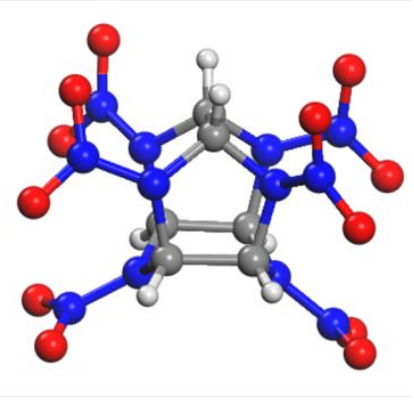

$\beta-C L-20$

Figure S4. Molecular configurations of CL-20 in the cocrystal 1 and cocrystal 2 


\section{SI 4. Detonation property calculations}

The detonation velocities and detonation pressures for cocrystal 1 and cocrystal 2 were calculated via the linear output thermodynamic user-friendly software code. ${ }^{2}$ As a comparison, CL-20, 4,5-MDNI, and LLM-105 were also calculated. The calculated method is as follow:

$\mathrm{F}=100 \times \frac{n O+n N-\left(\frac{n H}{2 n O}\right)+\left(\frac{A}{3}\right)-\left(\frac{n B}{1.75}\right)-\left(\frac{n C}{2.5}\right)-\left(\frac{n D}{4}\right)-\left(\frac{n E}{5}\right)}{M W}-G$

$\mathrm{D}=\frac{F-0.26}{0.55}$

$\mathrm{P}=\rho_{0} D^{2}\left(1-0.713 \rho_{0}{ }^{0.07}\right)$

$\mathrm{F}, \mathrm{D}$, and $\mathrm{P}$ are the detonation factor, the detonation velocity, and the detonation pressure, respectively. Meanwhile, $\mathrm{nO}, \mathrm{nN}$, and $\mathrm{nH}$ are the number of oxygen, nitrogen, and hydrogen atoms in chemical formula. $\mathrm{nB}$ is the number of oxygen atoms in excess of those available to form $\mathrm{CO}_{2}$ and $\mathrm{H}_{2} \mathrm{O}$. $\mathrm{nC}$ is the number of oxygen atoms doubly bonded directly to carbon, as in carbonyl. $\mathrm{nD}$ is the number of oxygen atoms singly bonded directly to carbon, and $\mathrm{nE}$ is the number of nitro groups existing either as a nitrate ester configuration or as a nitric acid salt. $\mathrm{A}=1$ if the compound is aromatic, otherwise $A=0 . G=0.4$ for a liquid explosive and $G=0$ for a solid explosive. $\rho_{0}$ is the initial density of the unreacted explosive in $\mathrm{g} / \mathrm{cm}^{3}$.

The calculated detonation properties including detonation velocities and detonation pressures were listed in Table S3. 
Table S3. Predicted detonation performances of the two cocrystals, raw materials, and reference

\begin{tabular}{cccc}
\hline Sample & $\rho\left(\mathrm{g} / \mathrm{cm}^{3}\right)$ & $\mathrm{D}(\mathrm{m} / \mathrm{s})$ & $\mathrm{P}(\mathrm{GPa})$ \\
\hline cocrystal 1 & 1.813 & 8604 & 34.45 \\
cocrystal 2 & 1.882 & 8972 & 38.59 \\
CL-20 & 2.040 & 9386 & 45.02 \\
4,5-MDNI & 1.624 & 7455 & 23.68 \\
LLM-105 & 1.908 & 8450 & 34.60 \\
\hline
\end{tabular}




\section{SI 5. References}

(1) Yang, Z. W.; Wang, H. J.; Ma, Y.; Huang, Q.; Zhang, J. C.; Nie, F. D.; Zhang, J.

H.; Li, H. Z. Isomeric Cocrystals of CL-20: A Promising Strategy for Development of High-Performance Explosives. Cryst. Growth Des. 2018, 18, 6399-6403.

(2) Muthurajan, H.; Sivabalan, R.; Talawar, M. B.; Asthana, S. N. Computer simulation for prediction of performance and thermodynamic parameters of high energy materials. J. Hazard. Mater. 2004, 112, 17-33. 